\title{
Nuclear data needs for advanced reactor systems: a NEA Nuclear Science Committee initiative
}

\author{
M. Salvatores ${ }^{1,2}$, G. Aliberti ${ }^{1}$, G. Palmiotti ${ }^{1}$, D. Rochman ${ }^{3}$, P. Oblozinsky ${ }^{3}$, M. Hermann ${ }^{3}$, P. Talou ${ }^{4}$, T. Kawano ${ }^{4}$, \\ L. Leal ${ }^{5}$, A. Koning ${ }^{6}$, and I. Kodeli ${ }^{7}$ \\ 1 Argonne National Laboratory, Argonne, IL 60439, USA \\ 2 CEA Cadarache, 13108 Saint-Paul-lez-Durance, France \\ 3 Brookhaven National Laboratory, Upton, NY 11973-5000, USA \\ ${ }^{4}$ Los Alamos National Laboratory, Los Alamos, NM 87543, USA \\ 5 Oak Ridge National Laboratory, Oak Ridge, TN 37831-6171, USA \\ ${ }^{6}$ NRG-Petten, 1755 ZG Petten, The Netherlands \\ 7 NEA-Databank, Le Seine Saint-Germain, 92130 Issy-les-Moulineaux, France
}

\section{Introduction}

The Working Party on Evaluation Cooperation (WPEC) of the OECD Nuclear Energy Agency Nuclear Science Committee has established an International Subgroup to perform an activity in order to develop a systematic approach to define data needs for Gen-IV and, in general, for advanced reactor systems. A methodology, based on sensitivity analysis has been agreed and representative core configurations for Sodium, Gas and Lead cooled Fast Reactors (SFR, GFR, LFR) have been defined as well as a high burn-up VHTR and a high burn-up PWR. In the case of SFRs, both a TRU burner (called in fact SFR) and a core configuration with homogeneous recycling of not separated TRU (called EFR) have been considered.

The methodology, the systems considered and the sensitivity approach are consistent with the work reported in ref. [1]. For the present study, the approach has been extended to the ABTR Na-cooled core, recently studied within the GNEP initiative [2].

Sensitivity coefficients (in a 15 energy group structure) have been calculated at ANL with the ERANOS code system [3] for all reactors and for the parameters most sensitive to nuclear data uncertainties: Multiplication factor, Power peak, Burn-up $\Delta \mathrm{k} / \mathrm{k}$, Coolant void reactivity coefficient, Doppler reactivity coefficient, Nuclide density at end of cycle (transmutation potential), Neutron source at fuel fabrication, Dose in a repository.

\section{Covariance data}

Preliminary cross section covariances have been developed for the WPEC Subgroup at BNL for 45 out of 52 requested materials $[4,5]$. The cross section covariances were produced in 15- and 187-group representations as follows:

- 36 isotopes (O-16; F-19; Na-23; Al-27; Si-28; Cr-52; Fe-56,57; Ni-58; Zr-90,91,92,94; Er-166,167,168,170; Pb-206,207,208; Bi-209; U-233,234,236; Np-237, Pu238,240,241,242; Am-241,242m,243; Cm-242,243,244,
245) were evaluated using the BNL-LANL methodology, based on the ENDF/B-VII.0 library [6], the Atlas of Neutron Resonances [7], the nuclear model code EMPIRE [8] and the Bayesian code Kalman [9];

- 6 isotopes (Gd-155,156,157,158,160 and Th-232) were taken from ENDF/BVII.0;

- 3 isotopes (H-1, U-238 and $\mathrm{Pu}-239)$ were taken from JENDL-3.3.

Covariances for the average number of neutrons per fission, total nu-bar, are provided for 16 actinides identified as priority by the subgroup.

LANL has evaluated the covariance matrices for U-235, $\mathrm{U} 238$ and $\mathrm{Pu}-239$, in the fast energy region, using only differential measurements and nuclear model calculations. A generalized-least-squares technique is used to evaluate a global covariance matrix based solely on experimental differential information. Since nuclear model calculations are used to complement experimental data, a Kalman filter is then used to combine experimental data and model calculations covariance matrices. This procedure has been used for the three isotopes U-235, U-238, and $\mathrm{Pu}-239$, for the reaction cross sections of (n,fission), (n,capture), (n,total), (n,elastic), (n,inelastic), and (n,xn). The covariance matrices related to the average number of neutrons were obtained from experimental data only.

To complete these data, at ORNL resonance-parameter covariance evaluations were done for U-235, U-238, and Pu239 with the computer code SAMMY [10]. For U-235 the covariance evaluations were done in the resolved and unresolved energy regions whereas for U-238 and Pu-239 only the resolved resonance covariance evaluations were done. Experimental uncertainties are incorporated directly into the evaluation process in order to propagate them into the resonance parameter results [11].

Finally, covariance data files for $\mathrm{Pb}$ isotopes have been produced at NRG by a purely stochastic approach [12]. This is accomplished by subjecting the nuclear model code TALYS [13] to a Monte Carlo scheme for perturbing the input parameters of the various nuclear models, such as level densities, gamma-ray strength functions and the optical model. 
Table 1. Fast neutron systems: total uncertainties (\%).

\begin{tabular}{|c|c|c|c|c|c|c|c|c|c|}
\hline \multicolumn{2}{|c|}{ Reactor } & $\mathbf{k}_{\text {eff }}$ & Power Peak & Doppler & Void & Burnup [pcm] & Decay Heat & Dose & Neutronic Source \\
\hline \multirow{2}{*}{ ABTR } & PEC $^{(a)}$ & 1.96 & 0.6 & 6.4 & 12.5 & 97 & 0.1 & 0.1 & 0.5 \\
\cline { 2 - 10 } & BOLNA $^{(b)}$ & 0.92 & 0.3 & 4.4 & 6.0 & 52 & 0.2 & 0.1 & 0.5 \\
\hline \multirow{2}{*}{ SFR } & PEC & 1.66 & 0.5 & 6.0 & 23.4 & 234 & 0.3 & 0.2 & 0.9 \\
\cline { 2 - 10 } & BOLNA $^{*}$ EFR & 1.82 & 0.4 & 5.6 & 17.1 & 272 & 0.4 & 0.3 & 1.0 \\
\cline { 2 - 10 } & PEC & 1.57 & 1.1 & 5.1 & 12.1 & 989 & 2.3 & 1.7 & 6.0 \\
\hline \multirow{2}{*}{ GFR } & PEC & 1.18 & 1.2 & 3.8 & 7.8 & 871 & 2.4 & 1.2 & 6.6 \\
\cline { 2 - 10 } & BOLNA & 1.80 & 1.8 & 5.5 & 7.1 & 384 & 0.5 & 0.6 & 1.8 \\
\hline \multirow{2}{*}{ LFR } & PEC & 2.26 & 1.7 & 5.5 & 7.7 & 381 & 0.4 & 0.5 & 1.4 \\
\cline { 2 - 10 } & BOLNA & 1.43 & 0.6 & 4.8 & 20.6 & 258 & 0.5 & 0.5 & 1.1 \\
\hline
\end{tabular}

(a) Partial Energy Correlation as used in ref. [1].

(b) BNL_ORNL_LANL_NRG_ANL.

Table 2. High burn-up VHTR: uncertainties (\%).

\begin{tabular}{|c|c|c|c|c|c|c|c|c|c|c|}
\hline \multicolumn{1}{c|}{\begin{tabular}{|c|c|c|c|c|c|c|}
\hline \multicolumn{1}{c|}{} \\
BOC
\end{tabular}} & $\begin{array}{c}\mathbf{k}_{\text {eff }} \\
\text { EOC }\end{array}$ & $\begin{array}{c}\text { Peak } \\
\text { Power } \\
\text { BOC }\end{array}$ & $\begin{array}{c}\text { Peak } \\
\text { Power } \\
\text { EOC }\end{array}$ & $\begin{array}{c}\text { Doppler } \\
\text { BOC }\end{array}$ & $\begin{array}{c}\text { Doppler } \\
\text { EOC }\end{array}$ & $\begin{array}{c}\text { Burnup } \\
\text { [pcm] }\end{array}$ & $\begin{array}{c}\text { Decay } \\
\text { Heat }\end{array}$ & & Dose & $\begin{array}{c}\text { Neutr. } \\
\text { Source }\end{array}$ \\
\hline PEC & 0.58 & 1.07 & 1.9 & 2.1 & 3.4 & 5.6 & 1574 & 3.1 & 2.6 & 14.3 \\
\hline BOLNA & 0.53 & 0.46 & 1.0 & 1.1 & 1.7 & 2.0 & 530 & 1.4 & 1.0 & 5.9 \\
\hline
\end{tabular}

Table 3. High burn-up PWR: uncertainties (\%).

\begin{tabular}{|c|c|c|c|c|c|c|c|c|}
\cline { 2 - 9 } \multicolumn{1}{c|}{} & $\begin{array}{c}\mathbf{k}_{\text {eff }} \\
\text { BOC }\end{array}$ & $\begin{array}{c}\mathbf{k}_{\text {eff }} \\
\text { EOC }\end{array}$ & $\begin{array}{c}\text { Doppler } \\
\text { BOC }\end{array}$ & $\begin{array}{c}\text { Doppler } \\
\text { EOC }\end{array}$ & $\begin{array}{c}\text { Burnup } \\
\text { [pcm] }\end{array}$ & $\begin{array}{c}\text { Decay } \\
\text { Heat }\end{array}$ & Dose & $\begin{array}{c}\text { Neutronic } \\
\text { Source }\end{array}$ \\
\hline PEC & 0.52 & 1.27 & 3.1 & 4.6 & 2206 & 3.8 & 3.1 & 13.2 \\
\hline BOLNA & 0.51 & 0.74 & 1.4 & 1.9 & 851 & 1.5 & 1.0 & 5.2 \\
\hline
\end{tabular}

In summary, for the present study, all the available BNL data have been used, except the U-235, U-238 and Pu-239 data, which have been taken from the combined LANL/ORNL evaluation and the $\mathrm{Pb}$ isotope data, taken from the NRG evaluation. Missing data have been taken from the ANL estimated covariance data [1].

\section{Results}

A selection of the results (which will be fully documented in a separate Subgroup report) is shown in tables 1 to 7 . Table 1 gives a summary of the integral parameter uncertainties in fast reactors (FR), and tables 2 and 3 for the high burn-up PWR and VHTR respectively.

Uncertainties obtained with the present compilation of variance-covariance data (called BOLNA: BNL_ORNL_ LANL_NRG_ANL) are compared to the ones obtained in ref. [1] using a preliminary compilation (PEC), performed at ANL. Uncertainties on the FR $\mathrm{k}_{\text {eff }}$ are still very relevant and

Table 4. $\Delta \rho$ burn-up uncertainty breakdown into components [pcm].

\begin{tabular}{|l|c|c|c|c|c|c|}
\hline System $\rightarrow$ & \multirow{2}{*}{ SFR } & EFR & GFR & LFR & VHTR & PWR \\
\cline { 1 - 6 }$\quad \Delta \rho$ component & & & & & & \\
\hline Actinides & \pm 272 & \pm 871 & \pm 381 & \pm 198 & \pm 530 & \pm 851 \\
\hline Fission products & \pm 73 & \pm 755 & \pm 130 & \pm 76 & \pm 215 & \pm 244 \\
\hline Total & \pm 282 & \pm 1153 & \pm 402 & \pm 212 & \pm 572 & \pm 885 \\
\hline
\end{tabular}

Table 5. Fast reactor systems: uncertainties (\%) due to $\mathrm{Pu}$ isotope cross sections (BOLNA).

\begin{tabular}{|c|c|c|c|c|c|c|c|}
\cline { 2 - 8 } \multicolumn{2}{c|}{} & \multicolumn{1}{c|}{ ABTR } & \multicolumn{2}{c|}{ SFR } & \multicolumn{2}{|c|}{ GFR } & LFR \\
\hline \multirow{2}{*}{ Isotope } & Cross Section & $\mathbf{k}_{\text {eff }}$ & $\mathbf{k}_{\text {eff }}$ & Void & $\mathbf{k}_{\text {eff }}$ & Peak Power & $\mathbf{k}_{\text {eff }}$ \\
\hline \multirow{2}{*}{ Pu-238 } & $v$ & 0.01 & 0.34 & 0.44 & 0.15 & 0.03 & 0.23 \\
\cline { 2 - 8 } & $\sigma_{\text {fission }}$ & 0.01 & 0.53 & 2.90 & 0.20 & 0.06 & 0.34 \\
\hline \multirow{2}{*}{$\mathbf{P u - 2 3 9}$} & $\sigma_{\text {fission }}$ & 0.24 & 0.12 & 0.87 & 0.15 & 0.03 & 0.21 \\
\cline { 2 - 8 } & $\sigma_{\text {capture }}$ & 0.23 & 0.12 & 1.16 & 0.23 & 0.06 & 0.17 \\
\hline \multirow{3}{*}{$\mathbf{P u - 2 4 0}$} & $v$ & 0.08 & 0.39 & 2.18 & 0.20 & 0.06 & 0.33 \\
\cline { 2 - 8 } & $\sigma_{\text {fission }}$ & 0.09 & 0.44 & 2.60 & 0.23 & 0.08 & 0.35 \\
\cline { 2 - 8 } & $\sigma_{\text {capture }}$ & 0.06 & 0.31 & 1.80 & 0.17 & 0.06 & 0.27 \\
\hline \multirow{2}{*}{$\mathbf{P u - 2 4 1}$} & $\sigma_{\text {fission }}$ & 0.12 & 0.96 & 4.09 & 0.82 & 0.16 & 0.61 \\
\hline \multirow{2}{*}{$\mathbf{P u - 2 4 2}$} & $\sigma_{\text {fission }}$ & 0.01 & 0.36 & 2.46 & 0.21 & 0.08 & 0.17 \\
\cline { 2 - 8 } & $\sigma_{\text {capture }}$ & 0.01 & 0.17 & 2.21 & 0.17 & 0.05 & 0.08 \\
\hline
\end{tabular}

generally beyond design target accuracies, even if a general reduction with respect to the data obtained in ref. [1] is observed. The uncertainties shown for the reactivity loss due to burn-up account only for the heavy element component since individual fission product uncertainties are not generally available. In ref. [1], an "integral" estimation of the uncertainty on the capture and scattering components of a "lumped" fission product was used, i.e. $10 \%$ on the capture cross section and $20 \%$ on the total scattering cross section of a "lumped" fission product in a fast spectrum, and $2 \%$ on the capture cross section of a "lumped" fission product in a thermal spectrum. The contribution of the fission product uncertainty to the overall burn-up reactivity is significant only in the case of a fast reactors with an extended burn-up (as it is the case of EFR, see table 4). For that case, it would be valuable, to improve the uncertainty assessment, to have available the covariance data of the $\sim 20$ most important isotopes, in particular in the fast energy range.

As for the other integral parameters, the present results confirm a relatively small impact of data uncertainties on the power peak values (the highest impact being in the GFR case) and on the Doppler coefficient, in the hypothesis of no uncertainty on the temperature dependence of the resonance self-shielding effects. As for the void reactivity coefficients, the impact of nuclear data uncertainty can be not negligible in Na-cooled systems and could have some impact on current Na-void coefficient minimisation studies. 
Table 6. Fast reactor systems: uncertainties (\%) due to selected MA cross sections (BOLNA).

\begin{tabular}{|c|c|c|c|c|c|c|c|}
\hline \multirow{2}{*}{ Isotope } & \multirow{2}{*}{ Cross Section } & ABTR & \multicolumn{2}{|c|}{ SFR } & \multicolumn{2}{|c|}{ GFR } & LFR \\
\cline { 3 - 8 } & & $\mathbf{k}_{\text {eff }}$ & $\mathbf{k}_{\text {eff }}$ & Void & $\mathbf{k}_{\text {eff }}$ & Peak Power & $\mathbf{k}_{\text {eff }}$ \\
\hline Am-241 & $\sigma_{\text {fission }}$ & 0.01 & 0.08 & 0.43 & 0.24 & 0.13 & 0.06 \\
\hline $\mathbf{A m - 2 4 2 m}$ & $\sigma_{\text {fission }}$ & 0.00 & 0.73 & 3.70 & 0.01 & 0.01 & 0.07 \\
\hline $\mathbf{A m - 2 4 3}$ & $\sigma_{\text {fission }}$ & 0.00 & 0.04 & 0.25 & 0.04 & 0.02 & 0.02 \\
\hline $\mathbf{C m - 2 4 4}$ & $\sigma_{\text {fission }}$ & 0.00 & 0.39 & 2.95 & 0.13 & 0.08 & 0.16 \\
\hline $\mathbf{C m - 2 4 5}$ & $\sigma_{\text {fission }}$ & 0.00 & 0.39 & 0.95 & 0.12 & 0.10 & 0.22 \\
\hline
\end{tabular}

Table 7. Fast reactor systems: uncertainties (\%) due to inelastic and capture (BOLNA).

\begin{tabular}{|c|c|c|c|c|c|c|c|}
\hline \multirow{2}{*}{ Isotope } & \multirow{2}{*}{ Cross Section } & ABTR & \multicolumn{2}{|c|}{ SFR } & \multicolumn{2}{|c|}{ GFR } & LFR \\
\cline { 2 - 8 } & $\mathbf{k}_{\text {eff }}$ & $\mathbf{k}_{\text {eff }}$ & Void & $\mathbf{k}_{\text {eff }}$ & Peak Power & $\mathbf{k}_{\text {eff }}$ \\
\hline \multirow{2}{*}{$\mathbf{U}-238$} & $\sigma_{\text {inelastic }}$ & 0.69 & 0.23 & 1.96 & 1.41 & 1.54 & 0.73 \\
\cline { 2 - 8 } & $\sigma_{\text {capture }}$ & 0.26 & 0.07 & 1.24 & 0.41 & 0.30 & 0.25 \\
\hline $\mathbf{F e - 5 6}$ & $\sigma_{\text {inelastic }}$ & 0.24 & 0.53 & 4.14 & 0.00 & 0.00 & 0.24 \\
\hline $\mathbf{N a}$ & $\sigma_{\text {inelastic }}$ & 0.07 & 0.25 & 13.43 & 0.00 & 0.00 & 0.00 \\
\hline $\mathbf{S i - 2 8}$ & $\sigma_{\text {inelastic }}$ & 0.00 & 0.00 & 0.00 & 0.22 & 0.25 & 0.00 \\
\hline $\mathbf{C}$ & $\sigma_{\text {elastic }}$ & 0.00 & 0.00 & 0.00 & 0.31 & 0.28 & 0.00 \\
\hline $\mathbf{P b - 2 0 6}$ & $\sigma_{\text {inelastic }}$ & 0.00 & 0.00 & 0.00 & 0.00 & 0.00 & 0.18 \\
\hline $\mathbf{P b - 2 0 7}$ & $\sigma_{\text {inelastic }}$ & 0.00 & 0.00 & 0.00 & 0.00 & 0.00 & 0.16 \\
\hline $\mathbf{P b - 2 0 8}$ & $\sigma_{\text {elastic }}$ & 0.00 & 0.00 & 0.00 & 0.00 & 0.00 & 0.13 \\
\hline
\end{tabular}

Table 8. Uncertainty (\%) on Pu isotope density at end of cycle (case of EFR).

\begin{tabular}{|c|c|c|c|c|c|c|}
\hline \multicolumn{2}{|c|}{ Uncertainty on $\rightarrow$} & \multirow{2}{*}{ Pu238 } & \multirow{2}{*}{ Pu239 } & Pu240 & Pu241 & Pu242 \\
\hline \multirow{2}{|c|}{ U238 } & capture & - & 1.1 & 0.2 & 0.1 & - \\
\hline \multirow{2}{*}{ Pu238 } & capture & 1.7 & 0.1 & - & - & - \\
\cline { 2 - 7 } & fission & 4.6 & - & - & - & - \\
\hline \multirow{2}{*}{ Pu239 } & capture & - & 0.8 & 1.3 & 0.7 & 0.1 \\
\cline { 2 - 7 } & fission & - & 0.2 & - & - & - \\
\hline \multirow{2}{*}{ Pu240 } & capture & 0.2 & - & 1.5 & 6.0 & 1.0 \\
\cline { 2 - 7 } & fission & - & - & 0.8 & 0.4 & - \\
\hline \multirow{2}{*}{ Pu241 } & capture & - & - & - & 0.8 & 1.5 \\
\cline { 2 - 7 } & fission & 0.2 & - & - & 5.0 & 0.7 \\
\hline \multirow{2}{*}{ Pu242 } & capture & - & - & - & - & 3.9 \\
\cline { 2 - 7 } & fission & - & - & - & - & 2.2 \\
\hline \multirow{2}{*}{ Am241 } & capture & 1.3 & - & - & - & 0.2 \\
\cline { 2 - 7 } & fission & 0.2 & - & - & - & - \\
\hline Total & & $\mathbf{5 . 1}$ & $\mathbf{1 . 3}$ & $\mathbf{2 . 1}$ & $\mathbf{7 . 9}$ & $\mathbf{4 . 9}$ \\
\hline
\end{tabular}

In summary, most of the uncertainty values shown in table 1, although sometimes significant, would not in principle affect the pre-conceptual design of any of the FR systems considered. However, some conservatism which could be suggested by the results shown in the tables, can have some economic impact in later phases of the design, and new evaluation/experiment (differential or integral) could be well justified in order to reduce uncertainties and associated cost.

In order to point out potential high priority domains of investigation, we have summarized the major features of the uncertainty impact for FRs in tables 5 to 7.

One can point out three major data sources for the overall uncertainties:

1. the $\mathrm{Pu}$ isotopes (other than $\mathrm{Pu}$-239) major reactions (fission, capture and nu-bar), see table 5. In the case of
Table 9. Uncertainty (\%) on selected MA density at end of cycle (case of EFR).

\begin{tabular}{|c|c|c|c|c|c|c|}
\hline \multicolumn{2}{|c|}{ Uncertainty on $\rightarrow$} & \multirow{2}{*}{\begin{tabular}{c} 
Am241 \\
\hline \multirow{2}{*}{ due to }
\end{tabular}} & Am242m & Am243 & Cm244 & Cm245 \\
\hline \multirow{2}{*}{ Pu240 } & capture & 1.6 & 0.6 & 0.2 & - & - \\
\cline { 2 - 7 } & fission & 0.1 & - & - & - & - \\
\hline \multirow{2}{*}{ Pu241 } & capture & 0.2 & 0.1 & 0.4 & 0.1 & - \\
\cline { 2 - 7 } & fission & 1.2 & 0.4 & 0.1 & - & - \\
\hline \multirow{2}{*}{ Pu242 } & capture & - & - & 9.3 & 4.1 & 1.5 \\
\cline { 2 - 7 } & fission & - & - & 0.6 & 0.2 & - \\
\hline \multirow{2}{*}{ Am241 } & capture & 3.1 & 2.0 & 0.1 & - & - \\
\cline { 2 - 7 } & fission & 0.9 & 0.5 & - & - & - \\
\hline \multirow{2}{*}{ Am242m } & capture & - & 1.6 & 0.3 & 0.2 & 0.1 \\
\cline { 2 - 7 } & fission & - & 7.4 & 0.1 & - & - \\
\hline \multirow{2}{*}{ Am243 } & capture & - & - & 1.9 & 1.9 & 1.0 \\
\cline { 2 - 7 } & fission & - & - & 0.5 & 0.2 & 0.1 \\
\hline \multirow{2}{*}{ Cm244 } & capture & - & - & - & 1.8 & 7.2 \\
\cline { 2 - 7 } & fission & - & - & - & 6.0 & 2.8 \\
\hline \multirow{2}{*}{ Cm245 } & capture & - & - & - & - & 0.9 \\
\cline { 2 - 6 } & fission & - & - & - & - & 15.6 \\
\hline \multicolumn{2}{|c|}{ Total } & $\mathbf{3 . 8}$ & $\mathbf{7 . 8}$ & $\mathbf{9 . 5}$ & $\mathbf{7 . 8}$ & $\mathbf{1 7 . 6}$ \\
\hline
\end{tabular}

$\mathrm{Pu}-239$, the major impact is due to the capture cross section, since the uncertainties associated to this isotope and in particular to its fission cross section are now extremely reduced, i.e. most often well below $1 \%$;

2. selected Minor Actinide fission cross sections (see table 6), but only in TRU burner fast reactors like the SFR, which has a $15 \%$ MA content in the fuel;

3 . inelastic cross section data (see table 7), and most notably U-238, Fe-56 and Na-23 (in Na-cooled FRs).

Besides these three wide "categories" of uncertainty contributions, one should not neglect still some impact of the $\mathrm{U}-238$ capture, despite the very small uncertainty values of the present covariance data evaluation.

As for the uncertainties on the nuclide densities variation between beginning and end of cycle, the most relevant results are once more related to cases where the irradiation time is significant. Since the case of the EFR is the fast reactor case with the highest burn-up, we show results for $\mathrm{Pu}$ isotopes (table 8) and for selected minor actinides (table 9). These tables give the uncertainty on the nuclide density at end of cycle. In all cases, as expected, the uncertainties are due to the capture and fission cross sections of the very same isotopes. The impact of such uncertainties can have some relevance on mass flows and inventories in the fuel cycle.

As far as thermal neutron systems, relatively small uncertainties on integral parameters are observed, see table 10 , since very small uncertainty are assumed on the low energy data of U-235, U-238, and Pu-239 and also of the Pu-240 capture close to the first resonance. There is however a few significant contributions as, e.g., the $\mathrm{Pu}-241$ fission cross section uncertainty to the PWR end-of-cycle reactivity.

As for the energy break-down of the uncertainties, table 11 gives, as a typical example, the energy break-down in the case of the SFR $\mathrm{k}_{\text {eff }}$ of a few fission and capture contributions. The wide energy range $(\sim 5 \mathrm{MeV}-1 \mathrm{keV})$ of relevance is due to the variety of fast spectra considered. 
Table 10. Thermal systems: uncertainties (\%) due to selected isotopes and reactions (BOLNA).

\begin{tabular}{|c|c|c|c|}
\hline \multirow{2}{*}{ Isotope } & \multirow{2}{*}{ Cross Section } & PWR & VHTR \\
\cline { 3 - 4 } & & $\mathbf{k}_{\text {eff }}$ EOC & \multirow{2}{*}{$\mathbf{k}_{\text {eff }}$ EOC } \\
\hline $\mathbf{U - 2 3 5}$ & $v$ & 0.17 & 0.27 \\
\hline \multirow{2}{*}{$\mathbf{U}-238$} & $\sigma_{\text {inelastic }}$ & 0.17 & 0.00 \\
\cline { 2 - 4 } & $\sigma_{\text {capture }}$ & 0.26 & 0.19 \\
\hline \multirow{2}{*}{$\mathbf{P u - 2 3 9}$} & $\sigma_{\text {fission }}$ & 0.18 & 0.10 \\
\cline { 2 - 4 } & $\sigma_{\text {capture }}$ & 0.07 & 0.11 \\
\hline \multirow{2}{*}{$\mathbf{P u - 2 4 0}$} & $\sigma_{\text {capture }}$ & 0.12 & 0.06 \\
\hline \multirow{2}{*}{$\mathbf{P u - 2 4 1}$} & $\sigma_{\text {fission }}$ & 0.34 & 0.18 \\
\cline { 2 - 4 } & $\sigma_{\text {capture }}$ & 0.13 & 0.13 \\
\hline $\mathbf{O}$ & $\boldsymbol{\sigma}_{\text {abs }}$ & 0.43 & 0.01 \\
\hline
\end{tabular}

Table 11. SFR $\mathrm{k}_{\text {eff }}$ uncertainties (\%). Energy breakdown [pcm] for selected isotopes/reactions.

\begin{tabular}{|c|c|c|c|c|c|}
\hline Group & Energy $^{(a)}$ & $\begin{array}{c}\text { Pu-238 } \\
\sigma_{\text {fission }}\end{array}$ & $\begin{array}{c}\text { Pu-240 } \\
\sigma_{\text {capture }}\end{array}$ & $\begin{array}{c}\text { Pu-241 } \\
\boldsymbol{\sigma}_{\text {fission }}\end{array}$ & $\begin{array}{c}\mathbf{A m - 2 4 2 m} \\
\boldsymbol{\sigma}_{\text {fission }}\end{array}$ \\
\hline $\mathbf{1}$ & $\mathbf{1 9 . 6 ~ M e V}$ & 0.01 & 0.00 & 0.02 & 0.02 \\
\hline $\mathbf{2}$ & $\mathbf{6 . 0 7} \mathbf{M e V}$ & 0.18 & 0.03 & 0.10 & 0.12 \\
\hline $\mathbf{3}$ & $\mathbf{2 . 2 3 ~ M e V}$ & 0.23 & 0.05 & 0.26 & 0.15 \\
\hline $\mathbf{4}$ & $\mathbf{1 . 3 5} \mathbf{M e V}$ & 0.31 & 0.11 & 0.40 & 0.28 \\
\hline $\mathbf{5}$ & $\mathbf{4 9 8} \mathbf{~ k e V}$ & 0.28 & 0.14 & 0.47 & 0.39 \\
\hline $\mathbf{6}$ & $\mathbf{1 8 3} \mathbf{~ k e V}$ & 0.12 & 0.16 & 0.58 & 0.39 \\
\hline $\mathbf{7}$ & $\mathbf{6 7 . 4} \mathbf{~ k e V}$ & 0.07 & 0.13 & 0.29 & 0.28 \\
\hline $\mathbf{8}$ & $\mathbf{2 4 . 8} \mathbf{~ k e V}$ & 0.06 & 0.13 & 0.16 & 0.12 \\
\hline $\mathbf{9}$ & $\mathbf{9 . 1 2} \mathbf{~ k e V}$ & 0.03 & 0.05 & 0.10 & 0.08 \\
\hline $\mathbf{1 0}$ & $\mathbf{2 . 0 3} \mathbf{~ k e V}$ & 0.03 & 0.01 & 0.08 & 0.10 \\
\hline $\mathbf{1 1}$ & $\mathbf{4 5 4} \mathbf{e V}$ & 0.00 & 0.00 & 0.03 & 0.02 \\
\hline $\mathbf{1 2 - 1 5}$ & $\mathbf{2 2 . 6} \mathbf{~ e V}$ & 0.00 & 0.00 & 0.00 & 0.00 \\
\hline Total & & $\mathbf{0 . 5 3}$ & $\mathbf{0 . 3 1}$ & $\mathbf{0 . 9 6}$ & $\mathbf{0 . 7 3}$ \\
\hline
\end{tabular}

(a) Upper energy boundary.

Finally, the impact of the correlation (off diagonal) terms is significant, and of the same order of magnitude of what observed in ref. [1].

\section{Summary and perspectives}

The present results are of a very high relevance for future reactor systems feasibility studies, since for the first time, a scientifically based, even if yet preliminary, set of variancecovariance data is available to reactor system designers, which allows to establish reliable uncertainties on all reactor and fuel cycle design parameters. A companion paper at this conference [14] will show which accuracies are required to meet current design target accuracies, and which strategies can be used to meet them. One important point seems to be the shift of priority from the three major actinide fission data to their inelastic (in particular for U-238) and capture data (for Pu-239, and, at a lesser extent, for U-238. The case of U-235 capture data in the $\mathrm{keV}$ region is presently under investigation). Higher priority should also be given to higher $\mathrm{Pu}$ isotopes (and in particular to their fission data) and to selected coolant/structural material inelastic cross sections (e.g., Fe-56 and Na-23).
Minor actinide data play a significant role only for dedicated burner reactors (ADS or critical) with Conversion Ratio $\mathrm{CR}=0$ and a content of MA in the fuel of $50 \%$ or higher. For more conventional burners $(\mathrm{Pu} / \mathrm{Ma} \sim 5)$ and down to $\mathrm{CR} \sim 0.25$, only selected MA data require significant improvements.

Finally, a complementary re-assessment of the uncertainty on decay heat and on some other fuel cycle parameters for innovative systems will be performed as part of the future activity of the WPEC Subgroup.

\section{References}

1. G. Aliberti, G. Palmiotti, M. Salvatores, T.K. Kim, T.A. Taiwo, M. Anitescu, I. Kodeli, E. Sartori, J.C. Bosq, J. Tommasi, Nuclear data sensitivity, uncertainty and target accuracy assessment for future nuclear systems, Ann. Nucl. Energy 33, 700 (2006).

2. Y.I. Chang, P.J. Finck, C. Grandy, Advanced Burner Reactor Preconceptual Design Report, ANL-ABR-1 (Argonne National Laboratory, Sept. 2006).

3. G. Rimpault et al., The ERANOS Code and Data System for Fast Reactor Neutronic Analyses, in Proceedings of PHYSOR 2002 Conference, Seoul, South Korea, October 2002.

4. D. Rochman, M. Herman, P. Oblozinsky, S.F. Mughabghab, Preliminary cross section covariances for WPEC Subgroup 26, Tech. Rep. BNL-77407-2007-IR (Brookhaven National Laboratory, 2007).

5. D. Rochman, M. Herman, P. Oblozinsky, S.F. Mughabghab, Preliminary nu-bar covariances for 238; $242 P u$ and 242; 243; 244; $245 \mathrm{Cm}$, Tech. Rep. BNL-77407-2007-IR-Suppl.1 (Brookhaven National Laboratory, 2007).

6. M. Chadwick, P. Oblozinsky et al., ENDF/B-VII.O: Next generation evaluated nuclear data library for nuclear science and technology, Nucl. Data Sheets 107, 2931 (Dec. 2006).

7. S.F. Mughabghab, Atlas of Neutron Resonances: Resonance Parameters and Thermal Cross Sections (Elsevier, Amsterdam, 2006).

8. M. Herman, R. Capote, B. Carlson, P. Oblozinsky, M. Sin, A. Trkov, V. Zerkin, EMPIRE nuclear reaction model code, version 2.19 (Lodi). www.nndc.bnl.gov/empire219/ (March 2005).

9. T. Kawano, Tech. Rep. JAERI-Research 99-009 (JAERI, 1999).

10. N.M. Larson, Updated Users' Guide for SAMMY: Multilevel R-Matrix Fits to Neutron Data Using Bayes' Equations, ORNL/TM-9179/R7 (2007).

11. L. Leal, H. Derrien, N. Larson, G. Arbanas, R. Sayer, ORNL Methodology for Covariance Generation for Sensitivity/Uncertainty Analysis, to be presented at the International Conference on Nuclear Criticality Safety (St. Petersburg, Russia, May 2007).

12. A.J. Koning, Generating covariance data with nuclear models, in Proceedings of the International Workshop on Nuclear data Needs for Generation IV Nuclear energy systems, Antwerpen, April 5-7, 2005, edited by P. Rullhusen (World Scientific, 2006), p. 153.

13. A.J. Koning, S. Hilaire M.C. Duijvestijn, TALYS: Comprehensive nuclear reaction modeling, in Proceedings of the International Conference on Nuclear Data for Science and Technology - ND2004, Santa Fe, USA, Sep. 26-Oct. 1, 2004, AIP 769, edited by R.C. Haight, M.B. Chadwick, T. Kawano, P. Talou (2005), p. 1154.

14. M. Salvatores, G. Aliberti G. Palmiotti, The role of differential and integral experiments to meet requirements for improved nuclear data (these proceedings). 